\title{
Coherent Single Electron Spin Control in a Slanting Zeeman Field
}

\author{
Yasuhiro Tokura, ${ }^{1,2}$ Wilfred G. van der Wiel, ${ }^{3}$ Toshiaki Obata, ${ }^{2}$ and Seigo Tarucha ${ }^{2,4}$ \\ ${ }^{1}$ NTT Basic Research Laboratories, NTT Corporation, Atsugi-shi, Kanagawa 243-0198, Japan \\ ${ }^{2}$ Quantum Spin Information Project, ICORP, JST, Atsugi-shi, Kanagawa 243-0198, Japan \\ ${ }^{3}$ SRO NanoElectronics, MESA ${ }^{+}$Institute for NanoTechnology, University of Twente, P.O. Box 217, \\ 7500 AE Enschede, The Netherlands, \\ and PRESTO-JST, University of Tokyo, Hongo, Bunkyo-ku, Tokyo 113-8656, Japan \\ ${ }^{4}$ Department of Applied Physics, University of Tokyo, Hongo, Bunkyo-ku, Tokyo 113-0033, Japan
}

(Received 14 October 2005; published 30 January 2006)

\begin{abstract}
We consider a single electron in a 1D quantum dot with a static slanting Zeeman field. By combining the spin and orbital degrees of freedom of the electron, an effective quantum two-level (qubit) system is defined. This pseudospin can be coherently manipulated by the voltage applied to the gate electrodes, without the need for an external time-dependent magnetic field or spin-orbit coupling. Single-qubit rotations and the controlled-NOT operation can be realized. We estimated the relaxation $\left(T_{1}\right)$ and coherence $\left(T_{2}\right)$ times and the (tunable) quality factor. This scheme implies important experimental advantages for single electron spin control.
\end{abstract}

DOI: 10.1103/PhysRevLett.96.047202

Stimulated by electron-spin-based proposals for quantum computation [1], a growing interest has emerged in realizing the coherent manipulation of a single electron spin in a solid-state environment. The application of the electron's spin - rather than its charge - as a quantum bit (qubit) is motivated by its potentially long coherence time in solids and the fact that it comprises a natural two-level system. Single electron spin resonance (SESR) plays a key role in realizing electron-spin-qubit rotation. Importantly, SESR is also the prime tool for determining the single electron spin coherence time $T_{2}$ in confined solid-state systems such as quantum dots (QDs). The induced Rabi oscillations can be read out via electron transport [2] or optically [3], giving an estimate for $T_{2}$. SESR was detected in paramagnetic defects in silicon [4] and for nitrogen vacancies in diamond [5], but not in semiconductor QDs so far. Realizing SESR in QDs is hard, not least because of the necessary high-frequency $(\sim 10 \mathrm{GHz})$ magnetic field in a cryogenic $(\sim 100 \mathrm{mK})$ setup. Waveguides and microwave cavities as used in conventional ESR [6] cause serious heating, limiting the operation temperature to $\sim 1 \mathrm{~K}$. Ongoing work in our group focuses on generating ac magnetic fields by an on-chip microscopic coil [7].

In this Letter, we propose a new SESR scheme that eliminates the need for an externally applied ac magnetic field, and with the potential of very high and tunable quality factors. An ac voltage is applied to let an electron in a QD oscillate under a static slanting Zeeman field. This effectively provides the electron spin with the necessary time-dependent magnetic field. Note the analogy with the Stern-Gerlach experiment, where the spin and orbital degrees of freedom are coupled by employing an inhomogenous magnetic field. The spatial oscillation of the electron within the QD involves the hybridization of orbital states, as schematically depicted in Fig. 1(a) for the case of the two lowest orbital states, $n=1,2$. Charge qubits based on
PACS numbers: 85.75.- d, 03.67.Lx, 76.30.-v, 85.35.Gv

double QDs [8] offer great tunability, but suffer from short coherence times ( $\sim 1 \mathrm{~ns})$ [9]. Spin qubits, on the contrary, enable long coherence times $(\sim 1 \mu \mathrm{s})$ [10], but are much harder to control, as pointed out above. Here, we present a hybrid charge-spin system that is promising both in terms of tunability and coherence. Analogously, the combination of the flux and charge degrees of freedom has proved to be fruitful in superconducting qubits [11]. We stress that in our system spin-orbit (SO) coupling is not required, as opposed to earlier work on electron spin control based on $g$-tensor modulation [12] and on electric fields [13].

A possible realization of the system is presented in Fig. 2. A 1D conductor such as a carbon nanotube or semiconductor nanowire is gated by ferromagnetic electrodes that define both the tunnel barriers of the QD and the slanting magnetic field. Alternatively, the slanting Zeeman field could be provided by a static inhomogeneity in the nuclear spin polarization or in the $g$ factor. The total (a)

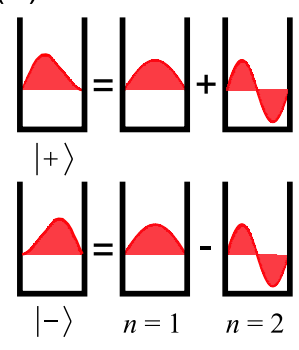

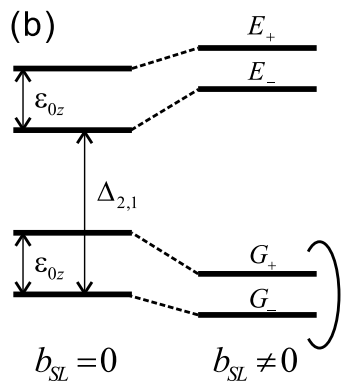

FIG. 1 (color online). (a) Schematic representation of how a spatial oscillation between wave functions $|+\rangle$ and $|-\rangle$ involves hybridization of multiple orbital states. (b) Energy spectrum of a quantum dot with two orbital levels (level spacing $\Delta_{2,1}$ ) and constant Zeeman energy $\varepsilon_{0 z}$ with or without a magnetic field gradient $b_{S L}$. The lowest levels, $\left|G_{ \pm}\right\rangle$, constitute a qubit. $\left|E_{ \pm}\right\rangle$ are excited states. 


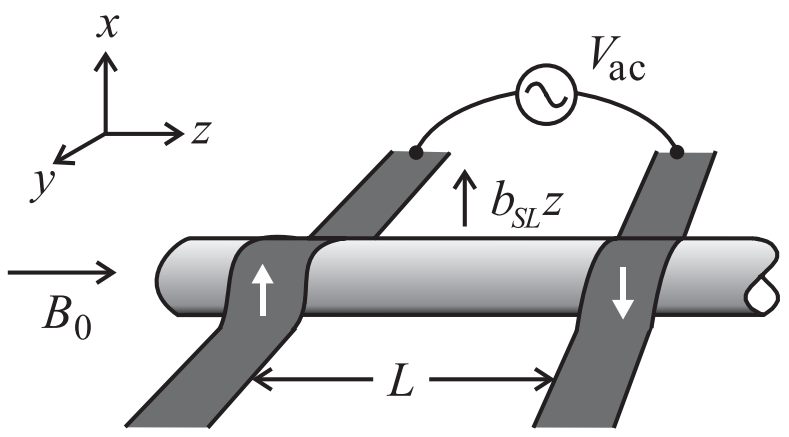

FIG. 2. Model of the 1D QD in a slanting Zeeman field. Ferromagnetic gate electrodes (dark gray) are located at either end of the dot and are magnetically polarized in the plus or minus $x$ direction, creating a magnetic field gradient $b_{S L}$. A uniform magnetic field $B_{0}$ is applied in the $z$ direction. The spin in the dot is controlled by applying an oscillating voltage $V_{\mathrm{ac}}$ between the two gates.

magnetic field is given by $\boldsymbol{B}=b_{S L} z \boldsymbol{i}_{x}+\left(B_{0}+b_{S L} x\right) \boldsymbol{i}_{z}$, where $B_{0}$ is the external uniform magnetic field parallel to the $z$ axis and $b_{S L}$ is the $z$-direction gradient of the field parallel to the $x$ axis (the middle of the QD corresponds to $z=0)$. A true 1D system is assumed with an electron strongly confined in the $x$ and $y$ directions. Therefore, the inhomogeneous term along the $z$ axis, $b_{S L} x \boldsymbol{i}_{z}$, can be eliminated (which is there to let $\boldsymbol{B}$ obey Maxwell's equations).

The Hamiltonian is $H_{0}=H_{00}+H_{0 s}$, where $H_{00}=$ $\frac{p_{z}^{2}}{2 m}+V(z)-g \mu_{B} B_{0} S_{z}$, and $H_{0 s}=-g \mu_{B} b_{S L} z S_{x}$, with $\boldsymbol{S}=\frac{1}{2} \boldsymbol{\sigma}, \boldsymbol{\sigma}$ the Pauli spin matrices, $g$ the effective $g$ factor, and $\mu_{B}$ the Bohr magneton [14]. $V(z)$ is the confinement potential of the QD with length $L$. The eigenvalues of $H_{00}$ are $\varepsilon_{n \sigma}=\varepsilon_{n}+\frac{1}{2} \varepsilon_{0 z} \sigma$, and the eigenfunctions $\langle z \mid n, \sigma\rangle=$ $\xi_{\sigma} \phi_{n}(z)$, where $n=1,2, \ldots, \sigma= \pm 1$, and $\xi_{\sigma}$ is the spinor. We define the Zeeman energy $\varepsilon_{0 z}=\left|g \mu_{B} B_{0}\right|$, which is assumed to be smaller than the orbital energy level separation $\Delta_{n, m}=\varepsilon_{n}-\varepsilon_{m} \ll U$, with $U$ the charging energy. The nonzero matrix elements of $H_{0 s}$ are $\left\langle m,-\sigma\left|H_{0 s}\right| n, \sigma\right\rangle \equiv \frac{1}{2} M_{m, n}$, with a coupling energy $M_{m, n} \equiv E_{S L} \Upsilon_{m, n}$, where $E_{S L} \equiv-g \mu_{B} b_{S L} L$ characterizing the strength of the slanting field, and the form factor $\Upsilon_{m, n} \equiv \int d z \phi_{m}^{*}(z) \frac{z}{L} \phi_{n}(z)$.

By requiring the confining potential to have a mirror symmetry, i.e., $V(z)=V(-z)$, the diagonal coupling energy elements vanish, namely, $M_{n, n}=0$. We employ perturbation theory up to the second order in $E_{S L}$ and obtain the ground-state energy for a pseudospin $\sigma, G_{\sigma}=\varepsilon_{1}+$ $\frac{1}{2} \varepsilon_{0 z} \sigma-\frac{1}{4} \sum_{l} \frac{M_{1,2 l}^{2}}{\Delta_{2 l, 1}-\varepsilon_{0 z} \sigma}$, and its wave function $\left|G_{\sigma}\right\rangle=$ $C_{\sigma}^{(0)}|1, \sigma\rangle+\sum_{l>0} C_{l \sigma}^{(1)}|2 l,-\sigma\rangle+\sum_{n>0} C_{n \sigma}^{(2)}|2 n+1, \sigma\rangle$ [15]. Since we assumed $\Delta_{2,1}>\varepsilon_{0 z}$, the two lowest energy states $\left|G_{+}\right\rangle$and $\left|G_{-}\right\rangle$represent an energetically isolated qubit (see Fig. 1). We can disregard higher energy states, such as $\left|E_{ \pm}\right\rangle$. For a rectangular confining potential, we find for the form factor $\Upsilon_{2 n+1,2 l}=-\frac{8}{\pi^{2}}(-1)^{l+n} \frac{2 l(1+2 n)}{\left[(1+2 n)^{2}-4 l^{2}\right]^{2}}$, while for a harmonic potential $V(z)=\frac{m \omega_{0}^{2}}{2} z^{2}$, we have $\Upsilon_{2 n+1,2 l}=\delta_{n+1, l} \sqrt{n+\frac{1}{2}}+\delta_{n, l} \sqrt{n}$, where we set $L=$ $\sqrt{\frac{\hbar}{m \omega_{0}}}$. Therefore, $\Upsilon_{n m}$ is negligible for large $|n-m|$, and we consider only $M_{1,2}$ and $M_{2,3}$, which is exact for the harmonic potential. We define the effective Zeeman energy $\varepsilon_{z} \equiv G_{+}-G_{-} \sim \varepsilon_{0 z}\left[1-\frac{1}{2} \frac{M_{1,2}^{2}}{\Delta_{2,1}^{2}-\varepsilon_{0 z}^{2}}\right]$.

We consider the qubit rotation induced by an ac electric field. The time-dependent perturbation $H_{1}(t)=e V_{\mathrm{ac}}(t) \frac{z}{L}$ is applied to the system by introducing an oscillating signal $V_{\text {ac }}(t)=V_{0} f(t)$ to the gate electrodes, as shown in Fig. 2. Since $H_{1}(t)$ is an odd function of $z$ and is independent of spin, only the off-diagonal matrix elements of $H_{1}$ remain, $\left\langle G_{\sigma}\left|H_{1}\right| G_{-\sigma}\right\rangle \sim\left(C_{1 \sigma}^{(1)}+C_{1-\sigma}^{(1)}\right) e V(t) \Upsilon_{21} \equiv \frac{1}{2} \varepsilon_{x} f(t)$, and the diagonal elements are zero for any order. Therefore, the effective Hamiltonian of our qubit is $H_{e}=$ $\frac{1}{2} \varepsilon_{z} \sigma_{z}+\frac{1}{2} \varepsilon_{x} f(t) \sigma_{x}$, which is formally equivalent to the conventional ESR Hamiltonian [16]. For a sinusoidal perturbation $f(t)=\cos \omega t$ at resonance $\left(\hbar \omega=\varepsilon_{z}\right)$, the time required for the $\pi$ operation, i.e., $\left|G_{+}\right\rangle \rightarrow\left|G_{-}\right\rangle$, is given by

$$
t_{\pi}=\frac{2 \pi \hbar}{\varepsilon_{x}} \sim \frac{\pi \hbar \Delta_{2,1}}{\Upsilon_{12}^{2} e V_{0}\left|E_{S L}\right|}\left(1-\frac{\varepsilon_{0 z}^{2}}{\Delta_{2,1}^{2}}\right) .
$$

Since we hybridize the spin and orbital degrees of freedom, orbital relaxation processes harm the spin coherence. We find that acoustic phonon scattering is the dominant relaxation mechanism for the energies relevant in our system. The electron-phonon scattering Hamiltonian is

$$
\begin{aligned}
H_{e-\mathrm{ph}} & =\sum_{q} \lambda_{q}\left(e^{i q z} b_{q}^{\dagger}+\text { H.c. }\right) \\
& =\sum_{q}\left(\Lambda_{q}^{x} \sigma_{x}+\Lambda_{q}^{z} \sigma_{z}\right) b_{q}^{\dagger}+\text { H.c., } \\
& \equiv \frac{1}{2} g \mu_{B}\left[B_{x}(t) \sigma_{x}+B_{z}(t) \sigma_{z}\right],
\end{aligned}
$$

where $q$ is the phonon wave number, $\lambda_{q}$ is the coupling constant, and $b_{q}^{\dagger}$ is the phonon creation operator. In Eq. (2), we project $H_{e-\text { ph }}$ to the qubit base, where the effective coupling constants are $\Lambda_{q}^{x}=\lambda_{q}\left\langle 1\left|e^{i q z}\right| 2\right\rangle\left(C_{1,+}^{(1)}+C_{1,-}^{(1)}\right)$ and $\Lambda_{q}^{z}=\lambda_{q}\left\langle 1\left|e^{i q z}\right| 3\right\rangle\left(C_{1,+}^{(2)}-C_{1,-}^{(2)}\right) . \quad B_{r}(t) \quad(r=x, y, z)$ represents the fluctuating magnetic field caused by phonons. A standard Born-Markov approximation [16] gives relaxation $\left(T_{1}\right)$ and coherence $\left(T_{2}\right)$ times as follows:

$$
\begin{gathered}
T_{1}^{-1}=k_{x x}\left(\varepsilon_{z}\right)+k_{y y}\left(\varepsilon_{z}\right), \\
T_{2 \text { phonon }}^{-1}=\left(2 T_{1}\right)^{-1}+k_{z z}(0),
\end{gathered}
$$

where $k_{r r^{\prime}}(\hbar \omega) \equiv \frac{1}{2}\left(g \mu_{B}\right)^{2} \int d \tau \cos (\omega \tau)\left\langle B_{r}(t) B_{r^{\prime}}(t+\tau)\right\rangle_{0}$ with $\langle\cdots\rangle_{0}$ representing the thermal average. The dephasing term $k_{z z}(0)$ related to $B_{z}(t) \sigma_{z}$ is negligible since 
$\left|\frac{\Lambda_{q}^{z}}{\Lambda_{q}^{x}}\right| \sim \frac{\varepsilon_{0 z} M_{2,3}}{\Delta_{3,1} \Delta_{2,1}} \ll 1$ and is shown to be absent in the zero frequency limit. This situation is similar to that of a spin qubit with SO interaction $[17,18]$. The relaxation rate $T_{1}^{-1}$ is then

$$
\begin{aligned}
T_{1}^{-1} & =\frac{2 \pi}{\hbar} \sum_{q}\left|\Lambda_{q}^{x}\right|^{2} \delta\left(\varepsilon_{z}-\hbar \omega_{q}\right) \operatorname{coth} \frac{\beta \varepsilon_{z}}{2} \\
& \sim\left(\frac{E_{S L} \Upsilon_{1,2} \Delta_{2,1}}{\Delta_{2,1}^{2}-\varepsilon_{0 z}^{2}}\right)^{2} \frac{1}{\tau_{p}\left(\varepsilon_{z}\right)},
\end{aligned}
$$

with $\hbar \omega_{q}$ the phonon energy, $\beta=1 /\left(k_{B} T\right)$, and the relaxation time $\tau_{p}(E)$ defined in analogy with Fermi's golden rule for a transition from level 2 to 1 with energy transfer $E$. The coherence time is obtained by

$$
T_{2 \text { total }}^{-1}=T_{2 \text { phonon }}^{-1}+T_{2 \text { spin }}^{-1}
$$

where we included the generic spin coherence time $T_{2 \text { spin }}$, which is the "pure" coherence time of the electron spin in the QD. The upper bound of the quality factor of the onequbit operation is characterized by $2 T_{1}$ divided by $t_{\pi}$ of Eq. (1),

$$
Q \sim \frac{2 \Delta_{2,1} \tau_{p}\left(\varepsilon_{z}\right)}{\pi \hbar} \frac{e V_{0}}{\left|E_{S L}\right|}\left(1-\frac{\varepsilon_{0 z}^{2}}{\Delta_{2,1}^{2}}\right)
$$

Importantly, the quality factor is tunable by controlling the amplitude of the ac voltage modulation, $V_{0}$.

For the practical implementation of our scheme, 1D systems with small electron-phonon coupling and/or weak SO coupling are favorable. Single wall carbon nanotube QDs are very suitable, because of the absence of piezoelectricity and the weak deformation potential coupling [19]. SO coupling does not play a role either. QDs in semiconductor (e.g., SiGe) nanowires are also good candidates, since $1 \mathrm{D}$ phonons couple weakly to the electron orbitals. Here we estimate $T_{1}$ and $Q$ of GaAs 1D QDs embedded in bulk AlGaAs. Since the phonon character is 3D in this system, the results are worse than for the more suitable systems given above. Figure 3(a) shows the lowtemperature $\left(\beta \varepsilon_{z} \gg 1\right)$ relaxation rate caused by bulk acoustic phonons in a QD with longitudinal parabolic confinement $\hbar \omega_{0}=1 \mathrm{meV}$ and a transversal confinement of $10 \mathrm{meV}$. $E_{S L}=1 \mu \mathrm{eV}$, corresponding to $b_{S L}=$ $1.16 \mathrm{~T} / \mu \mathrm{m}$, which can be realized with a ferromagnetic material [20]. Of the three acoustic phonon scattering mechanisms, transversal piezoelectric scattering is dominant for low $B_{0}$, where $T_{1}$ is of the order of $10 \mathrm{~ms}$. For comparison, the typical relaxation time from higher levels, i.e., $\left|E_{ \pm}\right\rangle \rightarrow\left|G_{ \pm}\right\rangle$in Fig. 1(b), is much shorter, $\sim 10 \mathrm{ns,}$ dominated by the deformation potential scattering. Note that the contribution of SO interaction (Dresselhaus coupling) is very small $\left(T_{2 \mathrm{SO}} \sim 10^{3} \mathrm{~s}\right)$ in $1 \mathrm{D}$ QDs, in contrast to disk-shaped dots as examined in Ref. [17]. Figure 3(b) shows $Q$ for various confinement potentials (dot length $L$ ) with $b_{S L}=1.16 \mathrm{~T} / \mu \mathrm{m}, V_{0}=10 \mu \mathrm{V}[21,22]$, and $t_{\pi} \sim$
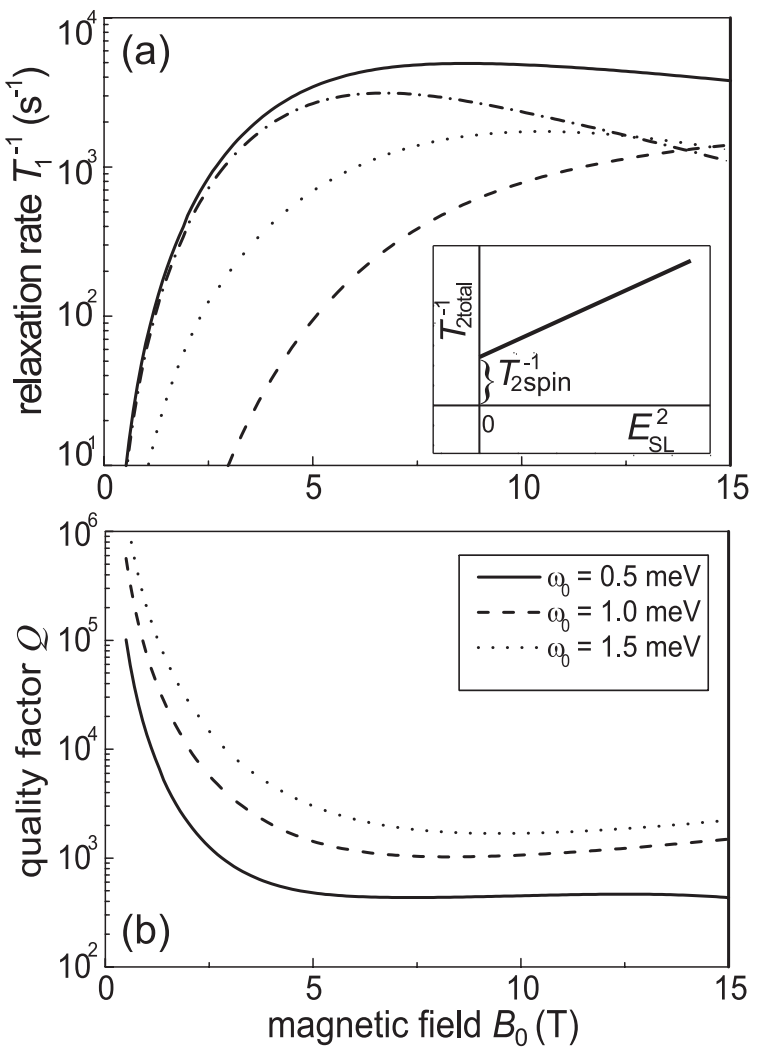

FIG. 3. (a) Relaxation rate $T_{1}^{-1}$ in a $1 \mathrm{D}$ GaAs QD as function of external magnetic field $B_{0}$ due to different phonon scattering mechanisms: deformation potential (dashed line), longitudinal piezoelectric (dotted line), transversal piezoelectric (dash-dotted line). The solid line is the total scattering rate. Inset: schematic derivation of the pure electron spin coherence time $T_{2 \text { spin }}$ from the dependence of the total coherence time $T_{2 \text { total }}$ on the strength of the slanting field $E_{S L}$. (b) $B_{0}$ dependence of the quality factor $Q$ for a single-qubit $\pi$ operation.

$400 \mathrm{~ns}$ for $\hbar \omega_{0}=1 \mathrm{meV}$. A quality factor $Q \gtrsim 10^{4}$ is often used as a threshold for viable quantum computation [23].

We study the time evolution of the density matrix of the four levels $\left|G_{ \pm}\right\rangle,\left|E_{ \pm}\right\rangle$, including $V_{\mathrm{ac}}(t)$ and phonon scattering. Near the resonant condition $\hbar \omega=\varepsilon_{z},\left|E_{ \pm}\right\rangle$are almost empty and do not contribute to the qubit dynamics at all. $T_{2 \text { total }}$ can be evaluated using a time-resolved measurement of the Rabi oscillation (see, e.g., [2]). After rotating the qubit over a certain angle, a projection measurement is done into $\left|G_{-}\right\rangle$or $\left|G_{+}\right\rangle$using a single-shot readout scheme based on spin-to-charge conversion [24]. Both energy selectivity and spin selectivity of tunneling out of the QD are applicable for our pseudospin qubit system. The readout error introduced by level mixing to $n=2$ by the slanting field is negligible, namely, of the order $\left(C_{1 \sigma}^{(1)}\right)^{2} \sim 10^{-6}$. Importantly, the pure electron spin coherence time $T_{2 \text { spin }}$ can be evaluated by extrapolating the $T_{2 \text { total }}$ dependence on $E_{S L}$ where $T_{2 \text { phonon }}^{-1} \propto E_{S L}^{2}$ [see Eq. (6)], as shown in the inset of Fig. 3(a). 
For a universal set of quantum gates, a two-qubit gate is required. Here we present a realization of a two-qubit gate based on two coupled dots in series [8]. Although it has been pointed out that an inhomogeneous magnetic field introduces swap errors $[25,26]$, we show that correct swap operation is possible in our system. The two-qubit Hamiltonian is $\mathcal{H}=\sum_{i=L, R} \mathcal{H}_{0 i}+\mathcal{H}_{T}+\mathcal{H}_{V}$, where $\mathcal{H}_{0 i}$ is the single-dot Hamiltonian, $i=L, R$ (ac field is off, $\left.\varepsilon_{x}=0\right), \mathcal{H}_{T}$ represents the tunneling between the dots, and $\mathcal{H}_{V}$ represents the interdot interaction $V$. By projecting the Hamiltonian onto the qubits, we find

$$
\begin{gathered}
\mathcal{H}_{0 i}=\frac{\varepsilon_{z}}{2} \sum_{\sigma} \sigma c_{i \sigma}^{\dagger} c_{i \sigma}+U n_{i \uparrow} n_{i \downarrow}, \\
\mathcal{H}_{T}=\sum_{\sigma}\left[t_{\sigma} c_{L \sigma}^{\dagger} c_{R \sigma}+s_{\sigma} c_{L \sigma}^{\dagger} c_{R-\sigma}+\text { H.c. }\right] \\
\mathcal{H}_{V}=V \sum_{\sigma \sigma^{\prime}} n_{L \sigma} n_{R \sigma^{\prime}},
\end{gathered}
$$

where $c_{i \sigma}$ annihilates an electron of pseudospin $\sigma$ in dot $i$. A spin-dependent tunneling term $t_{\sigma}$ and a tunneling term with spin flip $s_{\sigma}$ emerge, which are defined by $t_{\sigma}=$ $C_{\sigma}^{(0) 2} t_{11}+C_{1 \sigma}^{(1) L} C_{1 \sigma}^{(1) R} t_{22}+2 C_{1 \sigma}^{(2)} t_{13}, s_{\sigma}=\left(C_{1 \sigma}^{(1) L}+C_{1-\sigma}^{(1) R}\right) t_{12}$, where $t_{n m}$ represents the tunneling amplitude from level $n$ in $\operatorname{dot} L$ to level $m$ in dot $R$. The relevant lowest four eigenenergies and their eigenfunctions are obtained by the effective exchange Hamiltonian using local spin operators:

$$
\begin{aligned}
\mathcal{H}_{E X}= & J_{\|} S_{L z} S_{R z}+J_{\perp}\left(S_{L x} S_{R x}+S_{L y} S_{R y}\right) \\
& +\tilde{\varepsilon}_{z}\left(S_{L z}+S_{R z}\right),
\end{aligned}
$$

where $J_{\|}=\frac{2\left(t_{1}^{2}+t_{1}^{2}\right)}{U-V}-\frac{4 s^{2}(U-V)}{(U-V)^{2}-\varepsilon_{z}^{2}}, \quad J_{\perp}=\frac{4 t_{\uparrow} t_{\perp}}{U-V}, \quad$ and $\quad \tilde{\varepsilon}_{z}=$ $\varepsilon_{z}\left(1-\frac{2 s^{2}}{(U-V)^{2}-\varepsilon_{z}^{2}}\right)$ with $s=\frac{1}{2}\left(s_{\uparrow}+\frac{M_{1,2}^{R}}{M_{1,2}^{L}} s_{\downarrow}\right)$. It is well known that the SO interaction makes the exchange Hamiltonian anisotropic [27]. In contrast to the SO case, where the antisymmetric term dominates, the dominant anisotropic correction of $\mathcal{H}_{E X}$ in a slanting field is the symmetric term. Nevertheless, controlled-NOT operation can be accomplished by this anisotropic exchange Hamiltonian simply by replacing $J$ of the Heisenberg Hamiltonian by $J_{\|}$, and single-qubit operation (SESR) by replacing $\varepsilon_{0 z}$ by $\tilde{\varepsilon}_{z}$, as is shown in Refs. [26,27].

In conclusion, we propose a viable qubit based on combining the orbital and spin degrees of freedom of an electron in a QD placed in a slanting Zeeman field. Both single-qubit rotation and the controlled-NOT operation are demonstrated. This qubit is easier to manipulate than a spin qubit and has a better quality factor than a charge qubit. The concept is general and can be applied to a range of systems, such as single wall carbon nanotubes, GaAs, and $\mathrm{SiGe}$ QDs. This scheme also allows for the measurement of the intrinsic single electron spin coherence time.

We thank Y. Avishai, A. Khaetskii, and M. PioroLandrière for discussions. We acknowledge financial sup- port from DARPA Grant No. DAAD19-01-1-0659 of the QuIST program and SORST-JST.

[1] D. Loss and D.P. DiVincenzo, Phys. Rev. A 57, 120 (1998); R. Vrijen et al., Phys. Rev. A 62, 012306 (2000).

[2] H.-A. Engel and D. Loss, Phys. Rev. Lett. 86, 4648 (2001); Phys. Rev. B 65, 195321 (2002).

[3] O. Gywat et al., Phys. Rev. B 69, 205303 (2004); O. Gywat, H.-A. Engel, and D. Loss, J. Supercond. 18, 175 (2005).

[4] M. Xiao et al., Nature (London) 430, 435 (2004).

[5] F. Jelezko et al., Phys. Rev. Lett. 92, 076401 (2004).

[6] M. Dobers, K. von Klitzing, J. Schneider, G. Weimann, and K. Ploog, Phys. Rev. Lett. 61, 1650 (1988).

[7] T. Kodera et al., Physica (Amsterdam) 22E, 518 (2004); T. Kodera, W. G. van der Wiel, T. Maruyama, Y. Hirayama, and S. Tarucha, in Realizing Controllable Quantum States, edited by H. Takayanagi and J. Nitta (World Scientific Publishing, Singapore, 2005), pp. 445-450.

[8] W. G. van der Wiel et al., Rev. Mod. Phys. 75, 1 (2003).

[9] T. Hayashi et al., Phys. Rev. Lett. 91, 226804 (2003).

[10] J. R. Petta et al., Science 309, 2180 (2005).

[11] D. Vion et al., Science 296, 886 (2002).

[12] Y. Kato et al., Science 299, 1201 (2003).

[13] E. I. Rashba and Al. L. Efros, Phys. Rev. Lett. 91, 126405 (2003).

[14] L. S. Levitov and E. I. Rashba, Phys. Rev. B 67, 115324 (2003). The equivalent Hamiltonian is obtained for a 1D QD with a relatively weak Rashba SO interaction, namely, by taking the limit $\xi \rightarrow 0$ in Eq. (2) of this reference.

[15] The explicit forms of the coefficients $\operatorname{read} C_{\sigma}^{(0)}=$ $1-\frac{1}{8} \sum_{l} \frac{M_{1,2 l}^{2}}{\left(\Delta_{2 l, 1}-\varepsilon_{0 z} \sigma\right)^{2}}, \quad C_{l \sigma}^{(1)}=-\frac{1}{2} \frac{M_{1,2 l}}{\Delta_{2 l, 1}-\varepsilon_{0 z} \sigma}, \quad$ and $\quad C_{n \sigma}^{(2)}=$ $\frac{1}{4} \sum l \frac{M_{2 n+1,2 l} M_{2 l, 1}}{\Delta_{2 n+1,1}\left(\Delta_{2 l, 1}-\varepsilon_{0 z} \sigma\right)}$.

[16] C.P. Slichter, Principles of Magnetic Resonance (Springer-Verlag, Berlin, 1990).

[17] A. V. Khaetskii and Yu. V. Nazarov, Phys. Rev. B 64, 125316 (2001).

[18] V. N. Golovach, A. Khaetskii, and D. Loss, Phys. Rev. Lett. 93, 016601 (2004).

[19] H. Suzuura and T. Ando, Phys. Rev. B 65, 235412 (2002).

[20] J. Wróbel et al., Phys. Rev. Lett. 93, 246601 (2004).

[21] W. G. van der Wiel et al., Physica (Amsterdam) 272B, 31 (1999).

[22] W. G. van der Wiel et al., Photon Assisted Tunneling in Quantum Dots in Strongly Correlated Fermions and Bosons in Low-Dimensional Disordered Systems, edited by I. V. Lerner et al. (Kluwer, Dordecht, 2002), pp. 43-68.

[23] A. M. Steane, Phys. Rev. A 68, 042322 (2003), and references therein.

[24] J. M. Elzerman et al., Nature (London) 430, 431 (2004); R. Hanson et al., Phys. Rev. Lett. 94, 196802 (2005).

[25] R. de Sousa, X. Hu, and S. Das Sarma, Phys. Rev. A 64, 042307 (2001)

[26] X. Hu, R. de Sousa, and S. Das Sarma, Phys. Rev. Lett. 86, 918 (2001).

[27] G. Burkard and D. Loss, Phys. Rev. Lett. 88, 047903 (2002). 